\title{
PRESENCE OF ADRENAL LESIONS AT DIAGNOSIS OF CUSHING'S DISEASE
}

Moreno Moreno, P; Alhambra Expósito, MR; Muñoz Jiménez, C; Prior Sánchez, l; Gálvez Moreno, MA. Management Unit of Clinical Endocrinology and Nutrition. University Hospital Reina Sofía. Córdoba. Spain.

\section{OBJECTIVE}

Patients with Cushing's disease (CD) may have adrenal nodules or hyperplasia related chronic hyperstimulation by adrenocorticotropic hormone (ACTH). The aim of this study was to describe the prevalence of adrenal nodules or hyperplasia in patients with CD and their relation with ACTH levels at diagnosis.

\section{PATIENTS AND METHODS}

Descriptive study of patients with CD (1995-2015). Variables analyzed: age, sex, body mass index (BMI), ACTH, urinary-free cortisol (UFC), abdominal magnetic resonance (MRI) or computed tomography (CT). Statistical analysis: comparing proportions with the chisquared and comparing means with Student's test.

\section{RESULTS}

49 patients with Cushing Disease. $89,8 \%$ Women. $44,43 \pm 15,29$ years old. $63,6 \%$ in remission

33 patients with abdominal MRI or CT

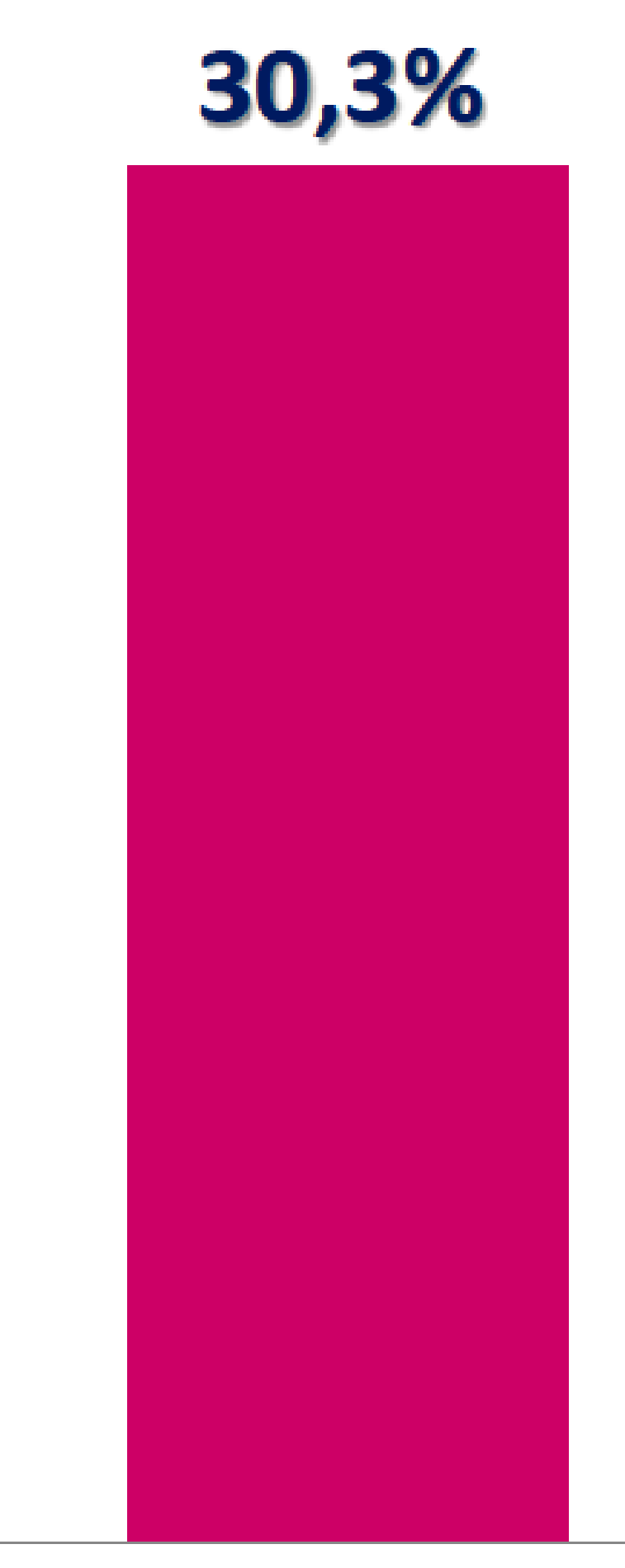

Adrenal nodules

\begin{tabular}{|c|c|c|c|}
\hline & $\begin{array}{c}\text { Adrenal lesions } \\
\text { (media } \pm \text { DE) }\end{array}$ & $\begin{array}{c}\text { No adrenal } \\
\text { lesions } \\
\text { (media } \pm \text { DE) }\end{array}$ & $p$ \\
\hline Age (years old) & $44,56 \pm 10,43$ & $43,81 \pm 18,9$ & 0,84 \\
\hline UFC $_{\text {(mcg/24h) }}$ & $636,89 \pm 675,26$ & $636,89 \pm 675,26$ & 0,61 \\
\hline ACTH $_{(\mathrm{pg} / \mathrm{ml})}$ & $80,42 \pm 60,80$ & $76,61 \pm 60,32$ & 0,87 \\
\hline BMI $_{(\mathrm{Kg} / \mathrm{m} 2)}$ & $31,90 \pm 10,24$ & $33,22 \pm 7,19$ & 0,66 \\
\hline
\end{tabular}

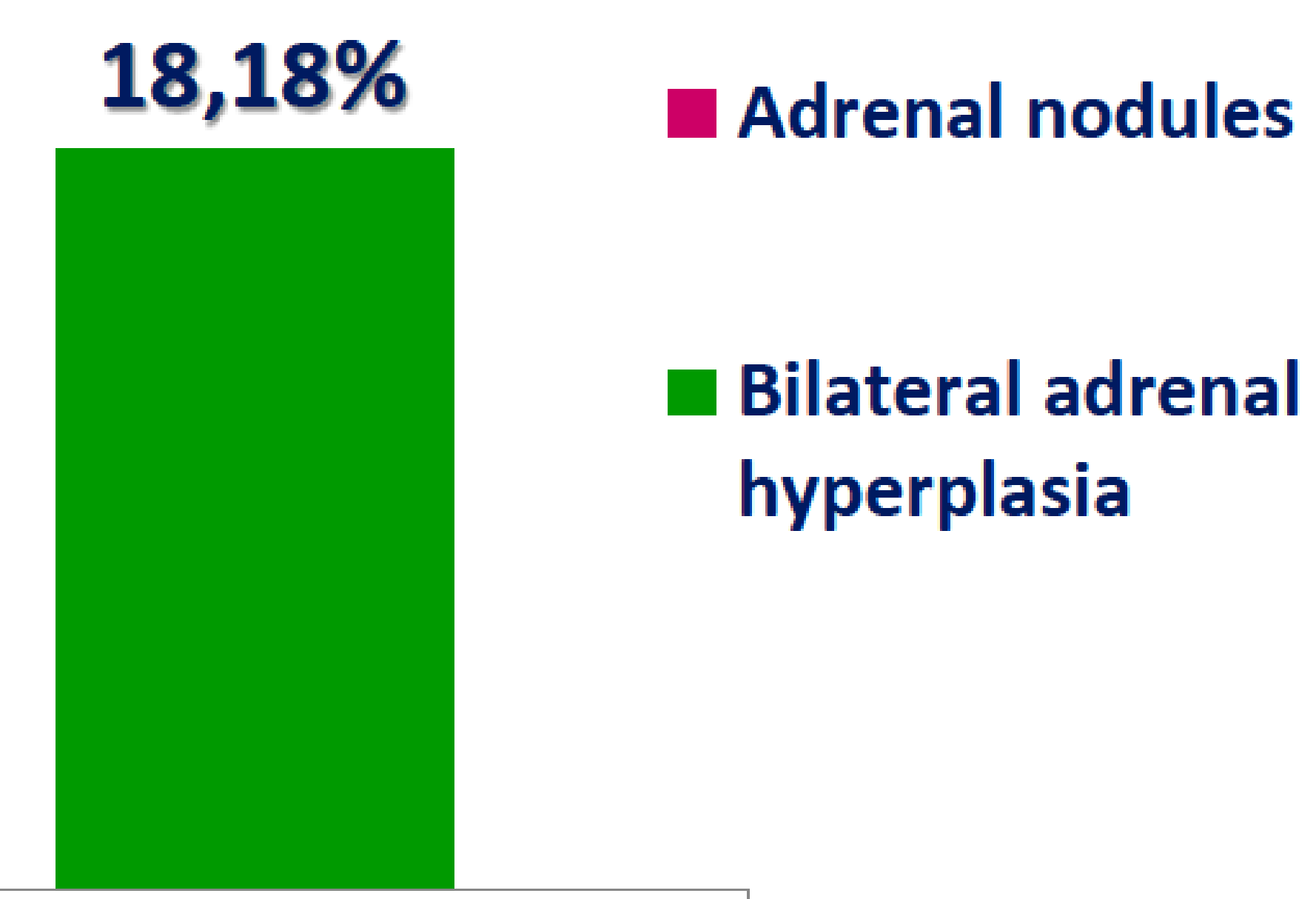

\begin{tabular}{|c|c|c|c|}
\hline & $\begin{array}{c}\text { Adrenal lesions } \\
n(\%)\end{array}$ & $\begin{array}{c}\text { No adrenal } \\
\text { lesions } \\
n(\%)\end{array}$ & $p$ \\
\hline Woman & $13(81,3)$ & $15(93,8)$ & 0,94 \\
\hline Remission of CD & $10(66,7)$ & $12(85,7)$ & 0,94 \\
\hline
\end{tabular}

\section{CONCLUSIONS}

Adrenal lesions are detected in half of the patients with CD which is performed abdominal MRI or TC, adrenal nodules are more frequent. The presence of adrenal lesions isn't related with ACTH levels at diagnosis, neither with UFC, BMI, age, sex or remission of $\mathrm{CD}$. 